\title{
OVERVIEW OF SATURN LIGHTNING OBSERVATIONS
}

\author{
G. Fischer*, U. A. Dyudina ${ }^{\dagger}$, W. S. Kurth ${ }^{\ddagger}$, D. A. Gurnett ${ }^{\ddagger}$, \\ P. Zarka ${ }^{\S}$, T. Barry $\mathbb{I}$, M. Delcroix ${ }^{\|}$, C. Go** D. Peach ${ }^{\dagger \dagger}$, \\ R. Vandebergh ${ }^{\ddagger}$, and A. Wesley
}

\begin{abstract}
The lightning activity in Saturn's atmosphere has been monitored by Cassini for more than six years. The continuous observations of the radio signatures called SEDs (Saturn Electrostatic Discharges) combine favorably with imaging observations of related cloud features as well as direct observations of flash-illuminated cloud tops. The Cassini RPWS (Radio and Plasma Wave Science) instrument and ISS (Imaging Science Subsystem) in orbit around Saturn also received ground-based support: The intense SED radio waves were also detected by the giant UTR-2 radio telescope, and committed amateurs observed SED-related white spots with their backyard optical telescopes. Furthermore, the Cassini VIMS (Visual and Infrared Mapping Spectrometer) and CIRS (Composite Infrared Spectrometer) instruments have provided some information on chemical constituents possibly created by the lightning discharges and transported upward to Saturn's upper atmosphere by vertical convection. In this paper we summarize the main results on Saturn lightning provided by this multi-instrumental approach and compare Saturn lightning to lightning on Jupiter and Earth.
\end{abstract}

\section{Radio Observations of SEDs by Cassini RPWS}

Saturn Electrostatic Discharges (SEDs) are short and strong radio bursts that were initially detected by the radio instrument on-board Voyager 1 near Saturn [Warwick et al.,

\footnotetext{
* Space Research Institute, Austrian Academy of Sciences, Schmiedlstrasse 6, A-8042 Graz, Austria

$\dagger$ California Institute of Technology, Pasadena, CA 91125 USA

‡Department of Physics and Astronomy, University of Iowa, Iowa City, IA 52242, USA

$\S$ LESIA, Observatoire de Paris, UMR CNRS 8109, 92195 Meudon, France

I Broken Hill (Barry) and Murrumbateman (Wesley), Australia

\| Commission des observations planétaires, Société Astronomique de France

** University of San Carlos, Philippines

${ }^{\dagger}{ }^{\dagger}$ British Astronomical Association, United Kingdom

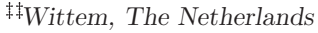


1981]. The SEDs were again detected by the Cassini RPWS instrument around Saturn Orbit Insertion [Fischer et al., 2006], and since then there has been practically continuous monitoring. Until the time of this writing the RPWS instrument has recorded 10 storms of Saturn lightning (named 0, A, B, C, D, E, F, G, H, and I) which are displayed in Figure 1 showing the number of SEDs per Saturn rotation as a function of time. Table 1 lists these SED storms giving their name, their start and stop days, the number of SEDs and episodes, and the re-occurrence period of the episodes. It is immediately obvious from Figure 1 that the SED activity in the last three years was particularly strong, whereas in the first three years of the Cassini mission the lightning activity was much more sparse. There was even an interval of 21 months from February 2006 until November 2007 with no SED activity. The periodic occurrence of great white spots with each Saturnian year [Sanchez-Lavega et al., 1991] suggests that the atmosphere of Saturn undergoes seasonal changes. It could be that the strong SED activity of the last years is linked to Saturn's equinox (August 11, 2009). Until the end of the Cassini mission in September 2017 the spacecraft will have stayed in Saturn's orbit for $\sim 45 \%$ of a Saturnian year. This and future ground-based optical and radio observations should enable the identification of a possible seasonality of Saturn lightning storms.

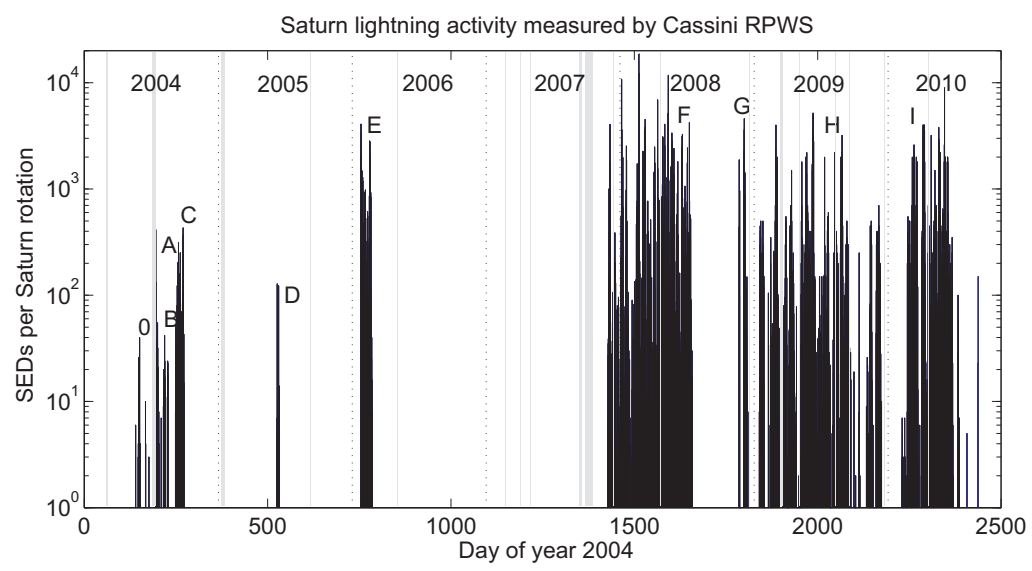

Figure 1: Number of SEDs recorded by Cassini/RPWS as a function of time. The gray background denotes data gaps, and the SED numbers from 2009 and later are estimates.

\section{Ground-based Radio Observations of SEDs}

SED signals are at least $10^{4}$ times stronger than the radio signals of terrestrial lightning in the frequency range of a few $\mathrm{MHz}$ [Fischer et al., 2006]. Several unsuccessful attempts were made to detect SEDs with ground-based radio telescopes. The negative results were either due to insufficient sensitivity or the intrinsic variability of SED occurrence [Zarka et al., 2008]. Finally, the high intensity of the SEDs and the real time information of Cassini about on-going lightning activity enabled the first convincing detection of SEDs by the 
Table 1: Saturn lightning storms recorded by Cassini/RPWS. The SED numbers give the number of detected SEDs plus the number of SED episodes (Saturn rotations during which SEDs were present). For the last two storms $H$ and I the exact numbers still have to be determined (TBD).

\begin{tabular}{|c|c|c|c|}
\hline Name & Start and stop date & SED numbers & Period \\
\hline 0 & May $26-31,2004$ & 100 in 8 & $10 \mathrm{~h} 35 \mathrm{~min}( \pm 6 \mathrm{~min})$ \\
$\mathrm{A}$ & July $13-27,2004$ & 800 in 15 & $10 \mathrm{~h} 43 \mathrm{~min}( \pm 3 \mathrm{~min})$ \\
$\mathrm{B}$ & August $3-15,2004$ & 300 in 16 & $10 \mathrm{~h} 40 \mathrm{~min}( \pm 3 \mathrm{~min})$ \\
C & Sept. $4-28,2004$ & 4,200 in 49 & $10 \mathrm{~h} 40 \mathrm{~min}( \pm 1 \mathrm{~min})$ \\
D & June $8-15,2005$ & 300 in 6 & $10 \mathrm{~h} 10 \mathrm{~min} ?$ \\
E & Jan. $23-$ Feb. 23,2006 & 43,400 in 71 & $10 \mathrm{~h} 40 \mathrm{~min}( \pm 0.4 \mathrm{~min})$ \\
F & Nov. $27,2007-$ July 15,2008 & 282,300 in 439 & $\sim 10 \mathrm{~h} 40 \mathrm{~min}$ \\
G & Nov. $19-$ Dec. 11, 2008 & 22,000 in 22 & $\sim 10 \mathrm{~h} 40 \mathrm{~min}$ \\
H & Jan. $14-$ Dec. 13,2009 & TBD in $\sim 470$ & $\sim 10 \mathrm{~h} 40 \mathrm{~min}$ \\
I & Feb. $7-$ July 14,2010 & TBD in $\sim 270$ & $\sim 10 \mathrm{~h} 40 \mathrm{~min}$ \\
\hline
\end{tabular}

large ground-based radio telescope UTR-2 in the Ukraine in early 2006 [Konovalenko et al., 2006]. For a detailed description we refer the reader to the article by Grießmeier et al. [2011] in this issue, and we only mention that SEDs are a good observational target for large radio telescopes like UTR-2 or LOFAR.

\section{Direct Optical Observations of Saturn Lightning Flashes}

The first direct optical observation of Saturn lightning by Cassini ISS occurred on August 17, 2009, and was recently published by Dyudina et al. [2010]. The reduced ring shine around equinox allowed the cameras to detect illuminated cloud tops on Saturn's night side. The size of the spots of a few hundred kilometers allowed the determination of the SED source depth which should be located 125-250 km below the cloud tops, most likely in the water cloud layer. From the brightness of the spots Dyudina et al. [2010] also inferred an optical energy of $\sim 10^{9} \mathrm{~J}$, which corresponds to the total energy of a terrestrial lightning flash. In Figure 2 we show another direct flash observation from November 30, 2009. As in August around equinox, the storm system was located at a planetocentric latitude of $35^{\circ}$ in the southern hemisphere, which at that time was still immersed in relative darkness (northern side of the rings illuminated). The storm clouds in Figure 2 and in all the other images of this paper should be due to moist convective storms that develop vertical convective plumes that overshoot the outermost ammonia $\left(\mathrm{NH}_{3}\right)$ cloud layer [Stoker, 1986; Hueso and Sanchez-Lavega, 2004].

\section{Optical Observations of Storm Clouds}

The first link between SEDs and optical observations of storm clouds was found in 2004, when the so-called "dragon storm" was imaged by Cassini ISS together with SED detec- 


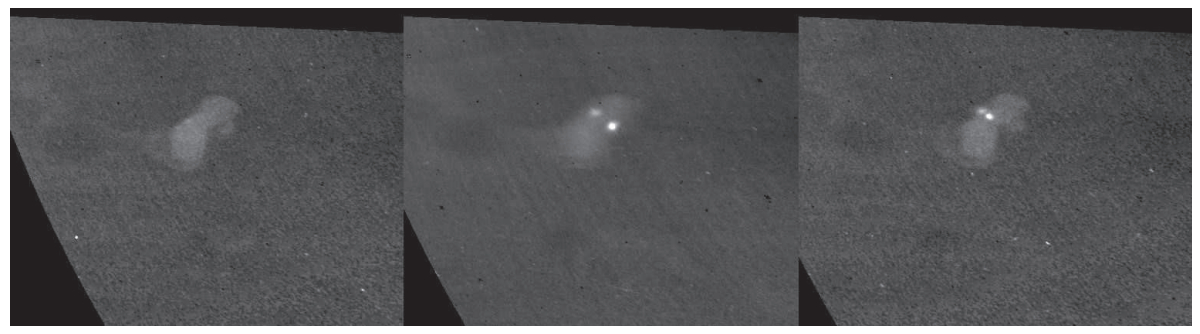

Figure 2: Sequence of three images by Cassini ISS from November 30, 2009, showing the SED storm cloud on the night side at $35^{\circ}$ south with lightning flashes (C)NASA).

tions by RPWS [Porco et al., 2005; Fischer et al., 2006]. These combined observations revealed consistent longitudes and longitudinal drift rates of the cloud feature with respect to the SED source. The white storm clouds were also found to be brighter when the SED rates were high [Dyudina et al., 2007; Fischer et al., 2007]. The "dragon storm" was located at a planetocentric latitude of $35^{\circ}$ south. This region is nicknamed "storm alley", and all SED storms shown in this section were observed there.

The involvement of amateur astronomers in the observation of Saturn's storms started with the one month long SED storm E in January/February 2006. It was the first longlasting SED storm observed by Cassini when Saturn was far from solar conjunction and thus high in the sky for ground-based observers. In the amateur's images the SED storms turned out to be detectable relatively easily, and they appear as bright white spots. We marked these spots with white arrows in the following figures since the loss in image quality by size reduction and printing makes some of them hard to discern. The left side of Figure 3 shows a sequence of 5 Saturn images taken by Ralf Vandebergh on February 2, 2006. It can be seen how a white spot at a latitude around $35^{\circ}$ south progressively comes into view from the right side, i.e. the western horizon since south is pointed upwards. Similarly, the sequence of 3 images on the right side of Figure 3 shows a storm system around the central meridian. It was taken nearly two years later by Marc Delcroix during SED storm $F$, and the tilt of the rings has decreased significantly.

Figure 4 shows two images from Cassini ISS taken during the 7.5 months long SED storm $\mathrm{F}$ which consisted of two phases. In the first phase from the end of November 2007 until early March 2008 only one storm system was present as imaged by Delcroix (right side of Figure 3) and by ISS (left side of Figure 4). From March 4-10, 2008, the RPWS instrument recorded uninterrupted SED activity for 9 consecutive Saturn rotations. This was a unique event since normally the SED activity is organized in episodes with periodic gaps when the storm is located beyond the radio horizon on the far side of the planet as seen from Cassini. The only explanation is that there was at least a second storm system present, and investigations by Delcroix and Fischer [2010] revealed that there were most likely even three storm systems at the same time in March 2008. After those 9 Saturn rotations the typical episodic behavior continued, but the longitude range at which SED activity was recorded seemed somewhat larger than usual. The reason for this was soon seen in the images, and this constitutes the second phase of SED storm F. A relatively persistent second storm system had developed which was located at the same latitude, 

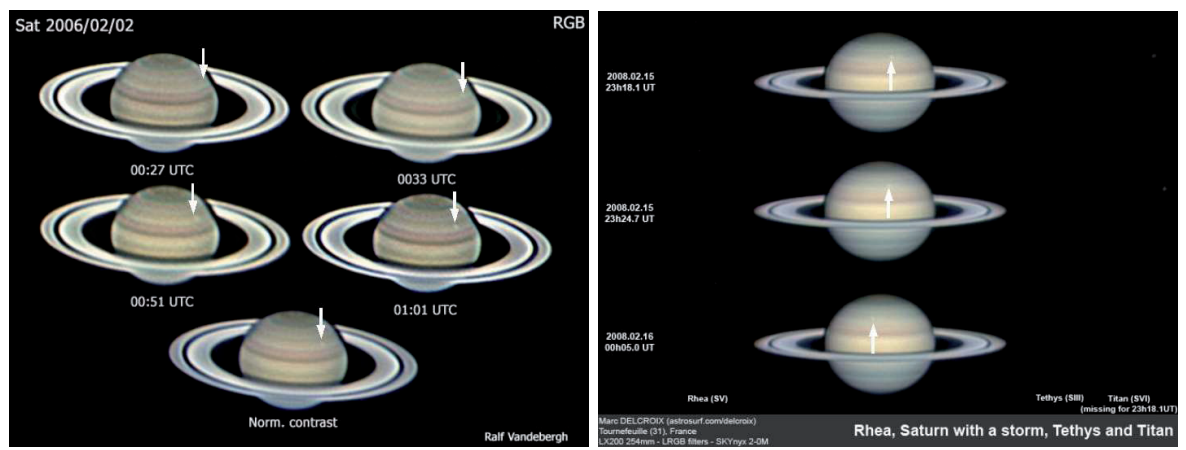

Figure 3: Saturn amateur images showing white spots around $35^{\circ}$ south during SED storms E and $F$, respectively. The sequence of 5 Saturn images on the left side was taken on February 2, 2006, by Ralf Vandebergh from the Netherlands. The sequence of 3 images on the right side was taken on February 15, 2008, by Marc Delcroix from France.

but the two storms were separated by about $30^{\circ}$ in longitude. The right side of Figure 4 clearly shows the two white clouds imaged by Cassini ISS on June 18, 2008. Groundbased observers even imaged them earlier, and the left side of Figure 5 shows an image with the two storms taken by Christopher Go on May 1, 2008.
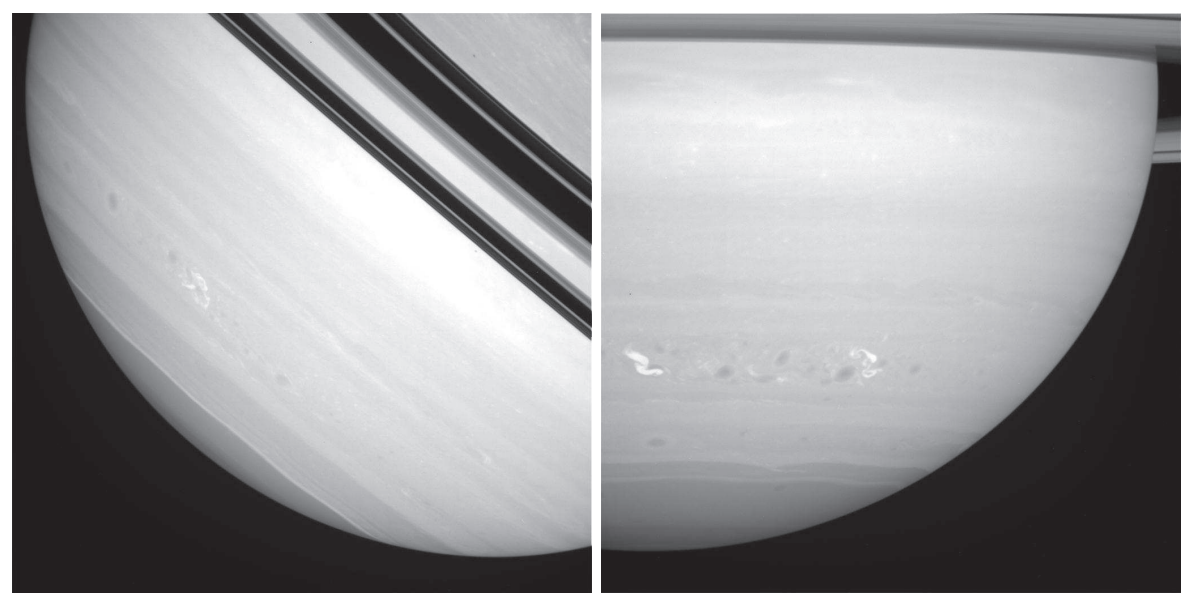

Figure 4: Cassini ISS images from March 4, 2008 (left), and June 18, 2008 (right side). The left image shows only one bright storm system, whereas there are two in the right image (CNASA).

The next SED storm G lasted only for about three weeks and took place in November/December 2008. Again, one white spot was seen at $35^{\circ}$ south as shown on the right side of Figure 5 in an image by Damian Peach (dark spot is the shadow of Dione). SED storm $\mathrm{G}$ was a kind of precursor to the next SED storm $\mathrm{H}$ that lasted from mid-January 

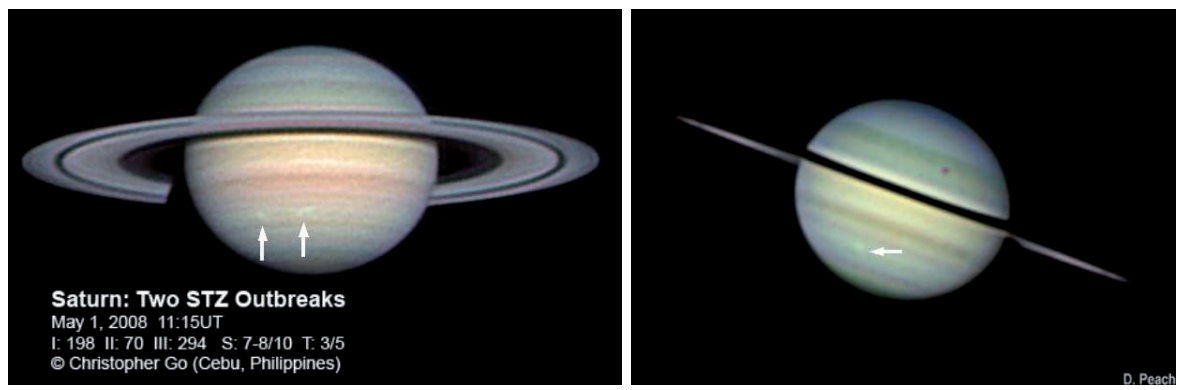

Figure 5: Saturn amateur images showing white spots around $35^{\circ}$ south during SED storm F and $G$, respectively. The image on the left side shows two storms and was taken on May 1, 2008, by Christopher Go from the Philippines. The right image was taken on December 7, 2008, by Damian Peach from the United Kingdom. South is downward in both images.

until mid-December 2009. With a duration of 11 months this SED storm is the longest ever observed lightning storm in our solar system to date. The left image of Figure 6 was taken by Anthony Wesley on March 6, 2009, with a white spot clearly visible around $35^{\circ}$ south in the storm alley. Another interesting feature of this image is the unusual brightness of Saturn's rings. This is the so-called Seeliger effect which enhances the brightness of reflective objects that are in opposition to the Sun. The image was taken just two days before the Earth moved through the line between Saturn and the Sun. The last image we show on the right side of Figure 6 was taken by Trevor Barry on June 3, 2010. Saturn had passed through equinox on August 11, 2009, and the northern side of the rings is illuminated in this image where south is pointing upwards. SED storm I of 2010 lasted for about 5 months, and like the other storms it started with a single white spot at $35^{\circ}$ south. However, several amateur images revealed a stretching of the storm in longitude with time. A few weeks before it faded away, there were actually three bright regions (marked by three white arrows) next to each other as it can be seen in the image by Barry.

In summary, for 8 of the 10 SED storms Cassini ISS and/or ground-based observers detected an associated cloud feature in the storm alley at a latitude of $35^{\circ}$ south. For only two SED storms (namely 0 and D, see Table 1) no associated storm cloud could be found, either due to the briefness and weakness of the SED storm or missing image coverage. These two storms occurred too close to solar conjunction to be observable from Earth. The ground-based optical observations of the amateurs have become increasingly important for Saturn lightning studies since Cassini ISS has lots of other observational targets. The major advantage of the RPWS instrument is the practically constant coverage, but the radio observations can only reveal an approximate location of the storms. The Cassini cameras can provide a very accurate positioning, but sometimes lack coverage. This is where the amateurs can help and during Saturn apparitions their coverage is usually good and sufficient for longitudinal drift rate measurements and observations of the storm shape evolution [Delcroix and Fischer, 2010]. Currently, there are more than 200 amateurs who have registered at the website of the PVOL, the Planetary Virtual Observatory 

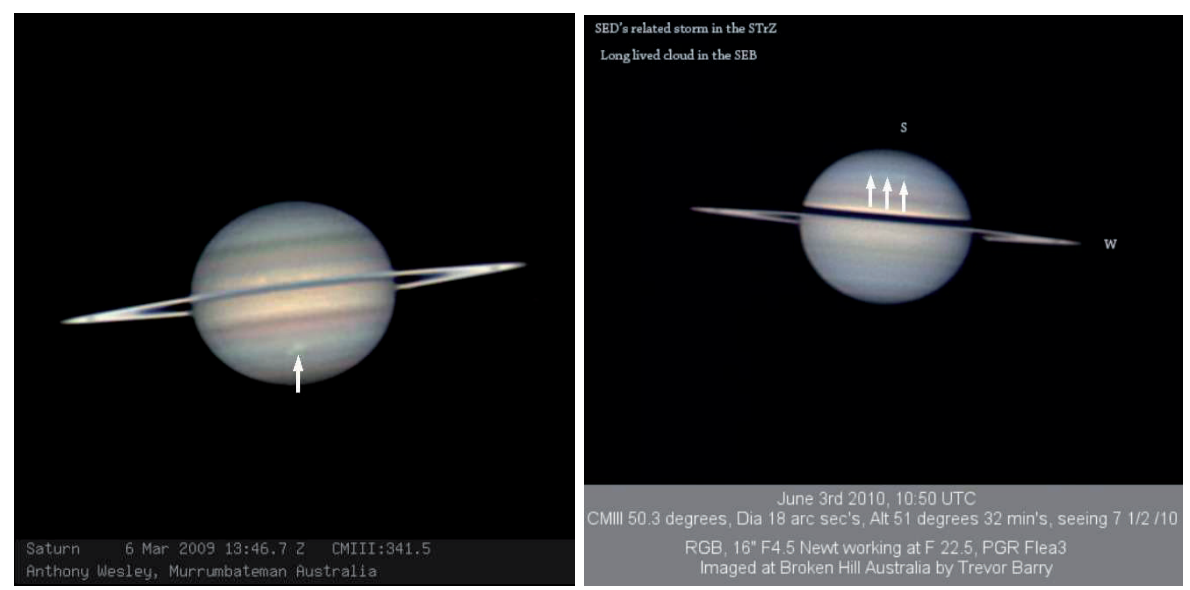

Figure 6: Saturn amateur images by the two Australians Anthony Wesley (left side, taken on March 6, 2009) and Trevor Barry (right side, taken on June 3, 2010). They show the white spots in Saturn's storm alley at $35^{\circ}$ south during SED storm $H$ and I, respectively. South is downward in the image by Wesley, but upward in the image by Barry.

and Laboratory at http://www.pvol.ehu.es/. This website was set up by the Planetary Sciences Group of the Universidad del País Vasco in Bilbao, Spain [Hueso et al., 2010]. Amateurs can post their planetary images there which can then be viewed by everyone. We inspected the catalog of Saturn images which goes back until 2001/2002. We found similar white spots in Saturn's southern storm alley during the Saturn apparitions of 2002/2003 and $2003 / 2004$, but none during $2001 / 2002$. It is very likely that these prominent white spots were also related to SED storms that could not be detected by the RPWS instrument due to the large distance of Cassini to Saturn at that time.

\section{Other Observations by Cassini VIMS and CIRS}

Bar-Nun [1975] predicted the existence of lighting on Jupiter prior to its detection by the Voyagers based on the presence of considerable acetylene $\left(\mathrm{C}_{2} \mathrm{H}_{2}\right)$ concentration. He first suggested that lightning may be the source of non-equilibrium concentration of various compounds in the atmospheres of the gas giants, and so new Cassini observations at Saturn are important to investigate lightning-induced atmospheric chemistry. The Cassini instruments VIMS and CIRS can observe Saturn's atmosphere at infrared wavelength and therefore reveal some chemical information. Spectroscopic observations by VIMS identified carbon soot particles attached to ammonia $\left(\mathrm{NH}_{3}\right)$ and ammonium hydrosulfide $\left(\mathrm{NH}_{4} \mathrm{SH}\right)$ condensates in dark spots close to Saturn's storm clouds [Baines et al., 2009]. The dark spots develop out of active lightning storms and usually drift to the west [Dyudina et al., 2007]. They are brought up to higher altitudes around 0.4 bar by vertical convection, and they can be seen at the same latitude like the white spots in both ISS 
images of Figure 4. Their dark color might come from the soot that is thought to be created by dissociation from methane $\left(\mathrm{CH}_{4}\right)$ in the high temperature lightning channel. In a news feature from April 29, 2010, published on the Cassini webpage, the CIRS team announced that it had found an enhanced level of phosphine $\left(\mathrm{PH}_{3}\right)$ potentially brought up to higher levels by convection. They also suspected that the lightning storm is similar to an ammonia-ice blizzard that is going on a few hundred kilometers below the tropopause.

\section{Comparison of lightning on Saturn, Jupiter, and Earth}

On Saturn lightning is intermittent, and usually there is only one large storm which can last for several months. On Jupiter there are typically a few storms at the same time, but they are about half the size of the Saturnian ones and only last for a few days. Terrestrial thunderstorms have an average duration of half an hour and an average size of $25 \mathrm{~km}$, but there are about 2000 thunderstorms in progress at any given moment [Rakov and Uman, 2003]. These and other characteristics are compared in Table 2.

Table 2: Comparison of lightning on Saturn, Jupiter, and Earth. The following references were used: ${ }^{a}$ Dyudina et al. [2007], ${ }^{b}$ Dyudina et al. [2010], ${ }^{c}$ Evans et al. [1983], ${ }^{d}$ Little et al. [1999], ${ }^{e}$ Lanzerotti et al. [1996], ${ }^{f}$ Rakov and Uman [2003], and ${ }^{g}$ Christian et al. [1989].

\begin{tabular}{|c|c|c|c|}
\hline Characteristic & Saturn & Jupiter & Earth \\
\hline Storm duration & days/months & days & tens of minutes/hours \\
Storm size & $a 2000-3000 \mathrm{~km}$ & $d<1500 \mathrm{~km}$ & $f 25 \mathrm{~km}$ \\
Illuminated cloud region & $b 100 \mathrm{~km}$ & $d 45-80 \mathrm{~km}$ & $g 10 \mathrm{~km}$ \\
Optical flash energy & $b 10^{9} \mathrm{~J}$ & $d 10^{9} \mathrm{~J}$ & $f 10^{6} \mathrm{~J}$ \\
Total flash energy & $10^{12} \mathrm{~J} ?$ & $10^{12} \mathrm{~J} ?$ & $f 10^{9} \mathrm{~J}$ \\
Stroke/flash duration & $c<140 \mu s ? / 70 \mathrm{~ms}$ & ${ }^{e} 500 \mu \mathrm{s} ? / ?$ & $f 70 \mu \mathrm{s} / 300 \mathrm{~ms}$ \\
\hline
\end{tabular}

For Saturn and Jupiter the illuminated cloud regions seen from above are the half width at half maximum (HWHM) of the flash brightness measured from the flash center. The larger size for Saturn is due to the greater depth of the lightning source below the cloud tops [Dyudina et al., 2010]. For Earth, illuminated cloud regions have a typical size of $10 \mathrm{~km}$, but can be up to $60 \mathrm{~km}$ for large storms [Christian et al., 1989]. The optical energies for Jovian and Saturnian flashes are similar $\left(\sim 10^{9} \mathrm{~J}\right)$, and about 3 orders of magnitude larger compared to a normal terrestrial flash (not a superbolt). Using an optical efficiency of $\sim 0.1 \%$ [Borucki and McKay, 1987], we estimate that the total energy of a lightning flash on Jupiter and Saturn is $\sim 10^{12} \mathrm{~J}$. In Table 2 we distinguish between stroke and flash duration since it is very likely that flashes on Jupiter and Saturn consist of sub-strokes. The Galileo Probe has measured waveforms of Jovian strokes [Lanzerotti et al., 1996], but it is unclear if they form a longer flash. Jovian lightning has no high frequency radio component detectable from space with which this question could be answered. For Saturn we probably have not yet resolved the sub-structure of the flashes. 


\section{Summary and Conclusions}

The Cassini RPWS instrument has continuously monitored the radio emissions of Saturn lightning during the last six years. This paper shows that studying Saturn lightning has become a multi-instrumental task, and other instruments have provided important new clues and additional information. Besides RPWS it involves the Cassini instruments ISS, VIMS, CIRS, as well as ground-based optical observations and measurements by giant radio telescopes. This fruitful cooperation should be continued in future and last at least until the end of the Cassini mission in 2017.

Acknowledgements. This work was supported by the Austrian Science Fund (FWF) in the frame of the project P21295-N16.

\section{References}

Baines, K. H., M.L. Delitsky, T. W. Momary, R. H. Browan, B. J. Buratti, R. N. Clark, and P.D. Nicholson, Storm clouds on Saturn: Lightning-induced chemistry and associated materials consistent with Cassini/VIMS spectra, Planet. Space Sci., 57, 1650-1658, 2009.

Bar-Nun, A., Thunderstorms on Jupiter, Icarus, 24, 86-94, 1975.

Borucki, W.J., and C.P. McKay, Optical efficiencies of lightning in planetary atmospheres, Nature, 328, 509-510, 1987.

Christian, H. J., R. J. Blakeslee, and S. J. Goodman, The detection of lightning from geostationary orbit, J. Geophys. Res., 94, D11, 13329-13337, 1989.

Delcroix, M., and G. Fischer, Contribution of amateur observations to Saturn storm studies, EPSC 2010-132 Abstracts Vol. 5, European Planetary Science Congress, Rome, Italy, 2010.

Dyudina, U. A., A. P. Ingersoll, S. P. Ewald, C. C. Porco, G. Fischer, W. S. Kurth, M. D. Desch, A. Del Genio, J. Barbara, and J. Ferrier, Lightning storms on Saturn observed by Cassini ISS and RPWS during 2004-2006, Icarus, 190, 545-555, 2007.

Dyudina, U.A., A. P. Ingersoll, S. P. Ewald, C.C. Porco, G. Fischer, W. S. Kurth, and R. A. West, Detection of visible lightning on Saturn, Geophys. Res. Lett., 37, L09205, 2010.

Evans, D. R., J. H. Romig, and J. W. Warwick, Saturn's Electrostatic Discharges: Properties and theoretical considerations, Icarus, 54, 267-279, 1983.

Fischer, G., M. D. Desch, P. Zarka, M. L. Kaiser, D. A. Gurnett, W. S. Kurth, W. Macher, H. O. Rucker, A. Lecacheux, W. M. Farrell, and B. Cecconi, Saturn lightning recorded by Cassini/RPWS in 2004, Icarus, 183, 135-152, 2006.

Fischer, G., W.S. Kurth, U. A. Dyudina, M. L. Kaiser, P. Zarka, A. Lecacheux, A. P. Ingersoll, and D. A. Gurnett, Analysis of a giant lightning storm on Saturn, Icarus, 190, 528-544, 2007. 
Grießmeier, J.-M., P. Zarka, A. A. Konovalenko, G. Fischer, V. V. Zakharenko, B. Ryabov, D. Vavriv, V. Ryabov, H. O. Rucker, and the Radio-Exopla Collaboration, Groundbased study of Saturn lightning, in Planetary Radio Emissions VII, H. O. Rucker, W.S. Kurth, P. Louarn, and G. Fischer (eds.), Austrian Academy of Sciences Press, Vienna, this issue, 145-154, 2011.

Hueso, R., and A. Sanchez-Lavega, A three-dimensional model of moist convection for the giant planets. II. Saturn's water and ammonia moist convective storms, Icarus, 172, 255-271, 2004.

Hueso, R., J. Legarreta, S. Pérez-Hoyos, J. F. Rojas, A. Sánchez-Lavega, and A. Morgado, The international outer planets watch atmospheres node database of giant-planet images, Planet. Space Sci., 58, 1152-1159, 2010.

Konovalenko, A. A., A. Lecacheux, H. O. Rucker, G. Fischer, E. P. Abranin, N. N. Kalinichenko, I. S. Falkovch, and K. M. Sidorchuk, Ground-based decameter wavelength observations of Saturn electrostatic discharges, European Planetary Science Congress, EPSC2006-A-00229, Berlin, 2006.

Lanzerotti, L. J., K. Rinnert, G. Dehmel, F. O. Gliem, E. P. Krider, M. A. Uman, and J. Bach, Radio frequency signals in Jupiter's atmosphere, Science, 272, 858-860, 1996.

Little, B., C.D. Anger, A. P. Ingersoll, A. R. Vasavada, D. A. Senske, H. H. Breneman, W. J. Borucki, and the Galileo SSI Team, Galileo images of lightning on Jupiter, Icarus, 142, 306-323, 1999.

Porco, C. C., et al. (34 co-authors), Cassini imaging science: Initial results on Saturn's atmosphere, Science, 307, 1243-1247, 2005.

Rakov, V.A., and M. A. Uman, Lightning: Physics and Effects, Cambridge Univ. Press, Cambridge, 2003.

Sanchez-Lavega, A., F. Colas, J. Lecacheux, P. Laques, D. Parker, and I. Miyazaki, The Great White Spot and disturbances in Saturn's equatorial atmosphere during 1990, Nature, 353, 397-401, 1991.

Stoker, C. R., Moist convection - A mechanism for producing the vertical structure of the Jovian equatorial plumes, Icarus, 76, 106-125, 1986.

Warwick, J. W., J. B. Pearce, D. R. Evans, T. D. Carr, J. J. Schauble, J. K. Alexander, M. L. Kaiser, M. D. Desch, B. M. Pedersen, A. Lecacheux, G. Daigne, A. Boischot, and C.H. Barrow, Planetary Radio Astronomy observations from Voyager 1 near Saturn, Science, 212, 239-243, 1981.

Zarka, P., W. M. Farrell, G. Fischer, and A. Konovalenko, Ground-based and space-based radio observations of planetary lightning, Space Sci. Rev., 137, 257-269, 2008. 